\title{
LARGE GROUPS AND THEIR PERIODIC QUOTIENTS
}

\author{
A. YU. OLSHANSKII AND D. V. OSIN
}

(Communicated by Jonathan I. Hall)

\begin{abstract}
We first give a short group theoretic proof of the following result of Lackenby. If $G$ is a large group, $H$ is a finite index subgroup of $G$ admitting an epimorphism onto a non-cyclic free group, and $g_{1}, \ldots, g_{k}$ are elements of $H$, then the quotient of $G$ by the normal subgroup generated by $g_{1}^{n}, \ldots, g_{k}^{n}$ is large for all but finitely many $n \in \mathbb{Z}$. In the second part of this note we use similar methods to show that for every infinite sequence of primes $\left(p_{1}, p_{2}, \ldots\right)$, there exists an infinite finitely generated periodic group $Q$ with descending normal series $Q=Q_{0} \triangleright Q_{1} \triangleright \ldots$, such that $\bigcap_{i} Q_{i}=\{1\}$ and $Q_{i-1} / Q_{i}$ is either trivial or abelian of exponent $p_{i}$.
\end{abstract}

\section{INTRODUCTION}

Recall that a group $G$ is large if some finite index subgroup of $G$ admits a surjective homomorphism onto a non-cyclic free group. In fact, it is easy to show that if $G$ is large, then some finite index normal subgroup of $G$ has a free non-cyclic quotient. Given a subset $S$ of a group $G$, we denote by $\langle\langle S\rangle\rangle^{G}$ the normal closure of $S$ in $G$. In the recent paper [7], Lackenby observed that adding higher powered relations preserves the largeness of a finitely generated group. More precisely, Lackenby proved the following (under the additional assumption that $G$ was finitely generated).

Theorem 1.1. Let $G$ be a large group, and $H$ a normal subgroup of $G$ of finite index admitting a surjective homomorphism onto a non-cyclic free group, $g_{1}, \ldots, g_{k}$ elements of $H$. Then the quotient group $G /\left\langle\left\langle g_{1}^{n}, \ldots, g_{k}^{n}\right\rangle{ }^{G}\right.$ is large for all but finitely many $n \in \mathbb{N}$.

As noted in [7], this theorem has interesting applications to Dehn surgery on 3 -manifolds. It is also interesting from the algebraic point of view since its iterated applications allow one to construct infinite finitely generated periodic groups. (This is similar to the idea suggested by Gromov in [5] and elaborated by the first author in [10] with the property of being large in place of the property of being nonelementary hyperbolic.)

The proof of Theorem 1.1 suggested in [7] essentially uses the deep theory related to property $(\tau)$ and homology growth developed by Lackenby in his recent papers

Received by the editors April 19, 2006.

2000 Mathematics Subject Classification. Primary 20F50, 20F05, 20 E26.

The first author was supported in part by the NSF grants DMS 0245600 and DMS 0455881.

The second author was supported in part by NSF grant DMS 0605093. Both authors were supported in part by the Russian Fund for Basic Research grant 05-01-00895. 
(see [7] and the references therein). In the first part of this note we provide a short group theoretic proof of Theorem 1.1. In the second part we apply our method to prove a modified version of Theorem 1.1 (see Lemma 3.2) and use this version to construct infinite finitely generated periodic groups with certain specific properties.

More precisely, let $\Pi=\left(p_{i}\right)$ be a (finite or infinite) sequence of primes. In this paper we say that a group $Q$ is a $\Pi$-graded group if $Q$ admits a normal series

$$
Q=Q_{0} \triangleright Q_{1} \triangleright \ldots,
$$

such that $\bigcap_{i} Q_{i}=\{1\}$ and $Q_{i-1} / Q_{i}$ is either trivial or abelian of exponent $p_{i}$. Note that all subgroups $Q_{i}$ have finite index in $Q$ whenever $Q$ is finitely generated.

Theorem 1.2. For any infinite sequence of primes $\Pi=\left(p_{i}\right)$, there exists a finitely generated infinite periodic П-graded group.

Observe that every finitely generated $\Pi$-graded group is residually finite and if $Q$ is a periodic $\Pi$-graded group, then every element $x \in Q$ has order $p_{j_{1}} \cdots p_{j_{k}}$, where $p_{j_{1}}, \ldots, p_{j_{m}}$ is a subsequence of $\Pi$ depending on $x$ (see Lemma 3.3). For instance, if $\Pi=(p, p, \ldots)$, we obtain an infinite finitely generated periodic residually finite $p$-group. Such groups were first constructed by Golod [3] and then by Aleshin [1], Grigorchuk [4], Gupta-Sidki [6], Sushchansky [12], and others. Note that all these groups are residually nilpotent.

On the other hand, Theorem 1.2 can be applied to find examples of a quite different nature. Recall that $S$ is a section of a group $Q$ if $S$ is a quotient group of a subgroup of $Q$. Recall also that an infinite group is called just infinite if all its proper quotients are finite.

Corollary 1.3. There exists a finitely generated periodic just infinite group $Q$ such that:

(1) Orders of elements of $Q$ are square free.

(2) Every section of $Q$ is residually finite. In particular, $Q$ is residually finite.

(3) If $S$ is a finite section of $Q$, then $S$ is solvable and all Sylow subgroups of $S$ are abelian. In particular, every nilpotent section of $Q$ is abelian.

We note that the first examples of finitely generated residually finite just infinite periodic groups were constructed in [4]. However, groups from [4] were $p$-groups. In particular they were residually nilpotent as well as Golod groups [3] (compare with (3)). To the best of our knowledge no examples of infinite finitely generated residually finite periodic groups satisfying any of the properties (1)-(3) were known until now.

\section{Proof of Theorem 1.1}

Throughout this paper $F$ denotes a non-abelian free group. We write $H \rightarrow F$ if a group $H$ admits an epimorphism onto some $F$. The main ingredient of our proof is the following.

Theorem 2.1 (Baumslag, Pride, [2]). Suppose that a group $G$ admits a presentation with $n \geq 2$ generators and at most $n-2$ relations. Then $G$ is large.

Remark 2.2. In fact, Baumslag and Pride proved even a stronger result. Under the assumptions of Theorem 2.1, they showed that for every sufficiently large $m \in \mathbb{N}$ there is a normal subgroup $H=H(m) \leq G$ such that $G / H \cong \mathbb{Z} / m \mathbb{Z}$ and $H \rightarrow F$ (see $[2])$. 
Given elements $g, t$ of a certain group, we use the notation $g^{t}$ to express $t^{-1} g t$ and $C_{G}(g)$ to denote the centralizer of $g$ in $G$. The proof of Theorem 1.1 is based on the following auxiliary results.

Lemma 2.3. Let $G$ be a group, and $N$ a normal subgroup of $G, g \in N$. Let $T$ denote a set of representatives of the right cosets of $C N$ in $G$, where $C$ is an arbitrary subgroup of $C_{G}(g)$. Then $\langle\langle g\rangle\rangle^{G}=\langle\langle Z\rangle\rangle^{N}$, where $Z=\left\{g^{t} \mid t \in T\right\}$.

Proof. It suffices to show that $g^{s} \in\langle\langle Z\rangle\rangle^{N}$ for every $s \in G$. To this end we note that $s=f h t$ for some $f \in C \leq C_{G}(g), h \in N$, and $t \in T$. Hence

$$
g^{s}=g^{f h t}=g^{h t}=g^{t h^{\prime}},
$$

where $h^{\prime}=h^{t} \in N$. Thus $g^{s} \in\langle\langle Z\rangle\rangle^{N}$.

Lemma 2.4. For any finite collection of non-trivial elements $g_{1}, \ldots, g_{k}$ of a free group $F$ and any number $m \in \mathbb{N}$, there exists $M \in \mathbb{N}$ with the following property. For every $q \geq M$, there is a finite index normal subgroup $N \triangleleft F$ such that $g_{1}^{s}, \ldots, g_{k}^{s} \notin N$ for $1 \leq s \leq m$, but $g_{1}^{q}, \ldots, g_{k}^{q} \in N$.

Proof. It suffices to prove the lemma in the case when $F$ is finitely generated. Let $a_{1}, \ldots, a_{r}$ be a basis of $F, A(\mathbb{Z}, r)$ (respectively $A\left(\mathbb{F}_{p}, r\right)$ ) the algebra of formal power series in non-commutative variables $x_{1}, \ldots, x_{r}$ over $\mathbb{Z}$ (respectively $\mathbb{F}_{p}$ ), $X$ (respectively $X_{p}$ ) the ideal of $A(\mathbb{Z}, r)$ (respectively $A\left(\mathbb{F}_{p}, r\right)$ ) generated by $x_{1}, \ldots, x_{r}$. It is well known that the subset $1+X$ of $A(\mathbb{Z}, r)$ forms a group with respect to multiplication, and the map $a_{i} \rightarrow 1+x_{i}, 1 \leq i \leq r$, extends to an embedding $F \rightarrow 1+X$ (see $[8]$ ).

Clearly there are $l, M_{0} \in \mathbb{N}$ such that for any prime $p \geq M_{0}$ the natural image of the set $S=\left\{g_{i}^{s} \mid 1 \leq i \leq k, 1 \leq s \leq m\right\}$ in $A\left(\mathbb{F}_{p}, r\right) / X_{p}^{l}$ does not contain 1. Without loss of generality we may assume $M_{0} \geq l$. Recall that $F$ is a residually finite $p$-group for any prime $p$. Hence for each prime $2 \leq p \leq M_{0}$, there is a subgroup $N_{p}$ of index $p^{j(p)}$ in $F$ for a certain $j(p) \in \mathbb{N}$ such that $S \cap N_{p}=\emptyset$. Set $M=\prod_{2 \leq p \leq M_{0}} p^{j(p)}$. Each number $q \geq M$ is divisible by either $p^{j(p)}$ for some prime $2 \leq p \leq M_{0}$, or by a prime $p>M_{0} \geq l$. In the first case, we set $N=N_{p}$. In the second case the inequality $p>l$ implies that the image of every element of $1+X$ in $A\left(\mathbb{F}_{p}, r\right) / X_{p}^{l}$ has order $p$. Therefore, we can set $N$ to be the kernel of the homomorphism of $F$ onto its image in $A\left(\mathbb{F}_{p}, r\right) / X_{p}^{l}$.

Lemma 2.5. Let $F$ be a free group of rank $r \geq 2, g_{1}, \ldots g_{k}$ arbitrary elements of $F$. Then $\bar{F}=F /\left\langle\left\langle g_{1}^{q}, \ldots, g_{k}^{q}\right\rangle\right\rangle{ }^{F}$ is large for all but finitely many $q \in \mathbb{N}$.

Proof. Without loss of generality we may assume that $g_{1}, \ldots, g_{k}$ are non-trivial. By Lemma 2.4, there exists $M \in \mathbb{N}$ such that for any $q \geq M$ there is a finite index normal subgroup $N \triangleleft F$ such that for all $i=1, \ldots, k, g_{i}^{s} \notin N$ if $s=1, \ldots, k$, but $g_{i}^{q} \in N$. Note that the image of $g_{i}$ in $F / N$ has order at least $k+1$. Therefore the index of $\left\langle g_{i}\right\rangle N$ in $F$ is at most $j /(k+1)$, where $j=[G: N]$.

According to Lemma 2.3, the image $\bar{N}$ of the subgroup $N$ in $\bar{F}$ is isomorphic to $N /\langle\langle Z\rangle\rangle^{N}$, where $Z=\bigcup_{i=1}^{k}\left\{\left(g_{i}^{q}\right)^{t} \mid t \in T_{i}\right\}$ and $T_{i}$ is a set of representatives of the right cosets of $\left\langle g_{i}\right\rangle N$ in $F$. Therefore, $\bar{N}$ admits a presentation with

$$
\operatorname{rank} N=1+(r-1) j \geq 1+j
$$


generators and the number of relations

$$
\operatorname{card} Z=\sum_{i=1}^{k} \operatorname{card} T_{i}=\sum_{i=1}^{k}\left[F:\left\langle g_{i}\right\rangle N\right] \leq \frac{k j}{k+1}<j .
$$

Hence $\bar{N}$ is large by Theorem 2.1. As $\bar{N}$ is of finite index in $\bar{F}$, the group $\bar{F}$ is also large.

Proof of Theorem 1.1. Let $H$ be a normal subgroup of finite index in $G$ that admits a homomorphism $\varepsilon: H \rightarrow F$ onto a non-cyclic free group. By Lemma 2.3, for every $n \in \mathbb{N}$, the image $\bar{H}$ of $H$ in $G /\left\langle\left\langle g_{1}^{n}, \ldots, g_{k}^{n}\right\rangle\right\rangle^{G}$ is isomorphic to $H /\left\langle\left\langle Z^{n}\right\rangle\right\rangle^{H}$, where $Z$ is some finite subset of $H$ consisting of conjugates of $g_{i}$ 's and $Z^{n}=\left\{z^{n} \mid z \in Z\right\}$. Thus $\bar{H}$ admits a surjective homomorphism onto $F /\left\langle\left\langle\varepsilon\left(Z^{n}\right)\right\rangle\right\rangle^{F}=F /\left\langle\left\langle\varepsilon(Z)^{n}\right\rangle\right\rangle^{F}$, which is large for all but finitely many $n$ according to Lemma 2.5 applied to the set $\varepsilon(Z)$. Hence so are $\bar{H}$ and $G /\left\langle\left\langle g_{1}^{n}, \ldots, g_{k}^{n}\right\rangle\right\rangle^{G}$.

\section{Constructing Periodic П-GRAded groups}

To each group $G$ and each sequence of primes $\Sigma=\left(q_{i}\right)$, we associate a sequence of characteristic subgroups $\delta_{i}^{\Sigma}(G)$ of $G$ defined by $\delta_{0}^{\Sigma}(G)=G$ and

$$
\delta_{i}^{\Sigma}(G)=\left[\delta_{i-1}^{\Sigma}(G), \delta_{i-1}^{\Sigma}(G)\right]\left(\delta_{i-1}^{\Sigma}(G)\right)^{q_{i}} .
$$

It is easy to prove by induction that these subgroups have finite index in $G$ whenever $G$ is finitely generated. We need an auxiliary lemma, which follows immediately from a result of Levi (see [9, Lemma 21.61]).

Lemma 3.1. For any finite subset $S$ of non-trivial elements of a free group $F$, there exists $D \in \mathbb{N}$ such that for any infinite sequence of primes $\Sigma$, we have $\delta_{d}^{\Sigma}(F) \cap S=\emptyset$ for all $d \geq D$.

Throughout the rest of this section we fix an arbitrary infinite sequence $\Pi=\left(p_{i}\right)$ of primes and denote by $\omega_{k}$ the subsequence $\left(p_{k+1}, p_{k+2}, \ldots\right)$ of $\Pi$.

Lemma 3.2. Let $G$ be a finitely generated group. Suppose that $\delta_{r}^{\Pi}(G) \rightarrow F$ for some $r$. Then for any element $g \in \delta_{r}^{\Pi}(G)$ there is $m \geq r$ such that if $g^{n} \in \delta_{m}^{\Pi}(G)$, then $\left.\delta_{m}^{\Pi}(G) /\left\langle g^{n}\right\rangle\right\rangle^{G} \rightarrow F$.

Proof. By Lemma 2.3, $\left.\delta_{r}^{\Pi}(G) /\left\langle g^{n}\right\rangle\right\rangle^{G}$ is isomorphic to $\delta_{r}^{\Pi}(G) /\left\langle\left\langle h_{1}^{n}, \ldots, h_{k}^{n}\right\rangle\right\rangle_{r}^{\Pi}(G)$, where $h_{1}, \ldots, h_{k}$ are some conjugates of $g$. Thus $\left.\delta_{r}^{\Pi}(G) /\left\langle g^{n}\right\rangle\right\rangle^{G}$ surjects onto $\bar{F}=F /\left\langle\left\langle g_{1}^{n}, \ldots, g_{k}^{n}\right\rangle\right\rangle^{F}$, where $g_{i}$ stands for the image of $h_{i}$ in $F$. Without loss of generality we may assume that $g_{1}, \ldots, g_{k}$ are non-trivial. By Lemma 3.1, there exists $d$ such that $g_{i}^{s} \notin \delta_{d}^{\omega_{r}}(F)$ for $1 \leq i, s \leq k$. We denote the subgroup $\delta_{d}^{\omega_{r}}(F)$ by $N$.

Assume that $g^{n} \in \delta_{r+d}^{\Pi}(G)$. Then $g_{i}^{n} \in N$ for all $i$. We now repeat word-forword the arguments from the proof of Lemma 2.5 and conclude that the image $\bar{N}$ of $N$ in $\bar{F}$ admits a presentation with 2 more generators than relations. According to Remark 2.2, there is a subgroup $M \triangleleft \bar{N}$ such that $\bar{N} / M$ is cyclic of order $p_{r+d+1} \cdots p_{r+d+c}$ for some $c \geq 1$ and $M \rightarrow F$. Clearly $\delta_{c+d}^{\omega_{r}}(\bar{N})$ is a finite index subgroup of $M$ and hence $\delta_{c+d}^{\omega_{r}}(\bar{N}) \rightarrow F$. It remains to note that $\delta_{r+d+c}^{\Pi}(G) /\left\langle\left\langle g^{n}\right\rangle\right\rangle^{G}$ surjects onto $\delta_{c+d}^{\omega_{r}}(\bar{N})$ and set $m=r+d+c$. 
Proof of Theorem 1.2. We enumerate all elements of the free group of rank $2 F=$ $\left\{f_{1}, f_{2}, \ldots\right\}$ and construct a sequence of quotients $G_{i}$ of $F$ as follows. Let $G_{0}=F$ and suppose that we have already constructed $G_{i}$ such that

$$
\delta_{r_{i}}^{\Pi}\left(G_{i}\right) \rightarrow F
$$

for some $r_{i} \in \mathbb{N}$. Also let $g_{i+1}$ denote the image of $\left(f_{i+1}\right)^{p_{1} p_{2} \cdots p_{r_{i}}}$ in $G_{i}$. Then $g_{i+1} \in$ $\delta_{r_{i}}^{\Pi}\left(G_{i}\right)$ and we can choose $r_{i+1}>r_{i}$ and $n=n(i) \in \mathbb{N}$ according to Lemma 3.2 so that $g_{i+1}^{n} \in \delta_{r_{i+1}}^{\Pi}\left(G_{i}\right)$ and $\delta_{r_{i+1}}^{\Pi}\left(G_{i}\right) /\left\langle\left\langle g_{i+1}^{n}\right\rangle\right)^{G_{i}} \rightarrow F$. We set $G_{i+1}=G_{i} /\left\langle\left\langle g_{i+1}^{n}\right\rangle\right\rangle^{G_{i}}$. Clearly $\delta_{r_{i+1}}^{\Pi}\left(G_{i+1}\right) \rightarrow F$. Observe also that (2) and inequality $r_{i+1}>r_{i}$ imply that $\delta_{r_{i+1}}^{\Pi}\left(G_{i}\right)$ is a proper subgroup of $\delta_{r_{i}}^{\Pi}\left(G_{i}\right)$. Therefore,

$$
\left|G_{i+1} / \delta_{r_{i+1}}^{\Pi}\left(G_{i+1}\right)\right|=\left|G_{i} / \delta_{r_{i+1}}^{\Pi}\left(G_{i}\right)\right|>\left|G_{i} / \delta_{r_{i}}^{\Pi}\left(G_{i}\right)\right| .
$$

Let $K_{i}$ denote the kernel of the natural homomorphism $F \rightarrow G_{i}$. Clearly $G=$ $F / \bigcup_{i=1}^{\infty} K_{i}$ is a periodic group. Further set $Q=G / \bigcap_{i=1}^{\infty} \delta_{r_{i}}^{\Pi}(G)$. Obviously $Q$ is a $\Pi$-graded group. To show that $Q$ is infinite we observe that $\operatorname{Ker}\left(G_{i} \rightarrow G\right) \leq \delta_{r_{i}}^{\Pi}\left(G_{i}\right)$ for every $i$. Hence

$$
G / \delta_{r_{i}}^{\Pi}(G) \cong G_{i} / \delta_{r_{i}}^{\Pi}\left(G_{i}\right) .
$$

Inequality (3) now implies $\left|G / \delta_{r_{i}}^{\Pi}(G)\right| \rightarrow \infty$ as $i \rightarrow \infty$. Therefore, the group $Q$ is infinite as it surjects onto $G / \delta_{r_{i}}^{\Pi}(G)$ for every $i$.

To derive Corollary 1.3 we first list some general properties of П-graded groups in the case when $\Pi$ consists of distinct primes.

Lemma 3.3. Suppose that all primes in the sequence $\Pi=\left(p_{1}, p_{2}, \ldots\right)$ are distinct and $Q$ is a periodic $\Pi$-graded group with normal series (1). Then the following holds:

(a) Every element $x \in Q$ has order $p_{j_{1}} \ldots p_{j_{k}}$, where $p_{j_{1}}, \ldots, p_{j_{k}}$ is a subsequence of $\Pi$. Moreover, if $x \in Q_{i}$, then $j_{1}>i$.

(b) Every subgroup of $Q$ is a $\Pi$-graded group.

(c) Every quotient $\bar{Q}$ of $Q$ is a $\Pi$-graded group.

(d) If $Q$ is finitely generated, then every section of $Q$ is residually finite.

(e) Every finite section of $Q$ is solvable. Every nilpotent section of $Q$ is abelian.

Proof. To prove (a) we note that for every element $x \in Q$, there exists $m$ such that $Q_{m} \cap\langle x\rangle=\{1\}$. Hence the order of $x$ in $Q$ coincides with the order of the image of $x$ in the $\Pi$-graded group $Q / Q_{m}$. Thus it suffices to prove (a) for $\Pi$-graded groups admitting finite series of type (1). This can easily be done by induction on the length of the series.

Assertion (b) is trivial. To prove (c) it suffices to note that the intersection $I$ of the images of subgroups $Q_{i}$ in $\bar{Q}$ is periodic, but $I$ cannot have elements of any prime order according to (a).

If $R$ is a subgroup of $Q$ and $\bar{R}$ is a quotient of $R$, then the same argument as above shows that the intersection of images $\bar{R}_{i}$ of $R_{i}=R \cap Q_{i}$ in $\bar{R}$ is trivial. Since $Q$ is finitely generated, $R / R_{i} \cong R Q_{i} / Q_{i} \leq Q / Q_{i}$ is finite. This proves (d) because $\left|\bar{R} / \bar{R}_{i}\right| \leq\left|R / R_{i}\right|$.

Note that every section of $Q$ is a $\Pi$-graded group by (b) and (c). Every finite $\Pi$-graded group admits a series (1) of finite length, hence every such group is solvable. Finally we recall that periodic nilpotent groups are locally finite and finite nilpotent groups are direct products of their Sylow subgroups. Obviously 
every finite $\Pi$-graded $p$-group is abelian whenever $\Pi$ consists of distinct primes. This implies (e).

Proof of Corollary 1.3. Suppose that $\Pi$ consists of distinct primes and $Q$ is a $\Pi$ graded group provided by Theorem 1.2. As $Q$ is finitely generated and infinite, by the Zorn Lemma there is a just infinite quotient $\bar{Q}$ of $Q$. The group $\bar{Q}$ is also a $\Pi$-graded group and have all required properties by Lemma 3.3.

Finally we mention one question motivated by Theorem 1.2 and Corollary 1.3. By $\mathcal{F S}$ we denote the class of all finite simple groups.

Problem 3.4. Does there exist an infinite finitely generated periodic group that is residually $\mathcal{F S}$ ?

Another interesting question is whether there exists an infinite finitely generated periodic residually finite group that is also residually simple. Problem 3.4 is related to one of the main open questions in the theory of hyperbolic groups, that is, whether every hyperbolic group is residually finite. If this question has a positive answer, our method can be used to construct finitely generated infinite periodic residually $\mathcal{F} \mathcal{S}$ groups. Here is the sketch of the proof.

In [11], the first author observed that if all hyperbolic groups are residually finite, then every non-elementary hyperbolic group has infinitely many finite simple quotients. Recall also that if $G$ is a non-elementary hyperbolic group and $g \in G$, then $G /\left\langle\left\langle g^{n}\right\rangle\right\rangle^{G}$ is also non-elementary hyperbolic provided $n$ is big enough [10]. Starting with a finitely generated non-abelian free group $F=\left\{f_{1}, f_{2}, \ldots\right\}$ and assuming that every hyperbolic group is residually finite, we can construct a sequence of non-elementary hyperbolic quotients $G_{i}$ of $F$ and subgroups $N_{i} \triangleleft G_{i}$ such that $G_{i+1}=G_{i} /\left\langle\left\langle g_{i+1}^{n}\right\rangle\right\rangle^{G_{i}}$ for some $n=n(i), G_{i} / N_{i}$ is finite simple, $\left|G_{i} / N_{i}\right| \rightarrow \infty$ when $i \rightarrow \infty$, and for every $i, g_{i+1}^{n}$ belongs to the intersection of the images of all subgroups $N_{0}, N_{1}, \ldots, N_{i}$ in $G_{i}$. Here $g_{i}$ is the image of $f_{i}$ in $G_{i}$ as above. Then we define the quotient group $G$ of $F$ as in the proof of Theorem 1.2 and denote by $Q$ the quotient of $G$ by the intersection of images of all subgroups $N_{i}, i \in \mathbb{N} \cup\{0\}$, in $G$. Clearly $Q$ is finitely generated, periodic, residually $\mathcal{F} \mathcal{S}$, and infinite for the same reason as above.

\section{REFERENCES}

[1] S. V. Aleshin, Finite automata and Burnside's problem on periodic groups, Math. Notes 11 (1972), no. 3, 199-203. MR0301107 (46:265)

[2] B. Baumslag and S. J. Pride, Groups with two more generators than relators, J. London Math. Soc. (2) 17 (1978), no. 3, 425-426. MR0491967 (58:11137)

[3] E. S. Golod, On nil-algebras and finitely approximable p-groups (Russian), Izv. Akad. Nauk SSSR, Ser. Mat. 28 (1964), 273-276. MR0161878 (28:5082)

[4] R. I. Grigorchuk, Degrees of growth of finitely generated groups and the theory of invariant means, Math. USSR-Izv. 25 (1985), no. 2, 259-300. MR764305 (86h:20041)

[5] M. Gromov, Hyperbolic groups, Essays in Group Theory, MSRI Series, Vol. 8, (S. M. Gersten, ed.), Springer, 1987, 75-263. MR919829 (89e:20070)

[6] N. Gupta and S. Sidki, On the Burnside problem for periodic groups, Math. Z. 182 (1983), 385-386. MR696534 (84g:20075)

[7] M. Lackenby, Adding high powered relations to large groups, prep., 2005; arXiv: math.GR/0512356.

[8] W. Magnus, A. Karras, and D. Solitar, Combinatorial group theory, Interscience Publ., 1966. MR2109550 (2005h:20052) 
[9] H. Neumann, Varieties of groups. Springer-Verlag New York, Inc., New York, 1967. MR0215899 (35:6734)

[10] A. Yu. Olshanskii, On residualing homomorphisms and $G$-subgroups of hyperbolic groups, Internat. J. Algebra Comput. 3 (1993), no. 4, 365-409. MR1250244 (94i:20069)

[11] A. Yu. Olshanskii, On the Bass-Lubotzky question about quotients of hyperbolic groups, $J$. Algebra 226 (2000), no. 2, 807-817. MR1752761 (2001i:20069)

[12] V. I. Suschansky, Periodic $p$-groups of permutations and the general Burnside problem, Dokl. Akad. Nauk SSSR 247 (1979), no. 3, 557-561. MR545692 (81k:20009)

Department of Mathematics, Vanderbilt University, Nashville, Tennessee 37240; And Department of Mathematics, Moscow State University, Moscow, 119899, Russia

E-mail address: alexander.olshanskiy@vanderbilt.edu

Department of Mathematics, The City College of New York, New York, New York 10031

E-mail address: denis.osin@gmail.com 